\title{
On a Li-type criterion for zero-free regions of certain Dirichlet series with real coefficients
}

\author{
Alina Bucur, Anne-Maria Ernvall-Hytönen, Almasa Odžak and Lejla Smajlović
}

\begin{abstract}
The $\mathrm{Li}$ coefficients $\lambda_{F}(n)$ of a zeta or $L$-function $F$ provide an equivalent criterion for the (generalized) Riemann hypothesis. In this paper we define these coefficients, and their generalizations, the $\tau$-Li coefficients, for a subclass of the extended Selberg class which is known to contain functions violating the Riemann hypothesis such as the Davenport-Heilbronn zeta function. The behavior of the $\tau$-Li coefficients varies depending on whether the function in question has any zeros in the half-plane $\operatorname{Re}(z)>\tau / 2$. We investigate analytically and numerically the behavior of these coefficients for such functions in both the $n$ and $\tau$ aspects.
\end{abstract}

\section{Introduction}

The extended Selberg class $\mathcal{S}^{\sharp}$, introduced in [10], consists of Dirichlet series $F$ satisfying the following axioms.

(i) (Dirichlet series) $F$ possesses a Dirichlet series representation

$$
F(s)=\sum_{n=1}^{\infty} \frac{a_{F}(n)}{n^{s}}
$$

that converges absolutely for $\operatorname{Re}(s)>1$.

(ii) (Analytic continuation) There exists an integer $m \geqslant 0$ such that $(s-1)^{m} F(s)$ is an entire function of finite order. The smallest such number is denoted by $m_{F}$ and called the polar order of $F$.

(iii) (Functional equation) The function

$$
\Phi_{F}(s)=F(s) Q_{F}^{s} \prod_{j=1}^{r} \Gamma\left(\lambda_{j} s+\mu_{j}\right),
$$

with $Q_{F}>0, r \geqslant 0, \lambda_{j}>0,|w|=1, \operatorname{Re}\left(\mu_{j}\right) \geqslant 0, j=1, \ldots, r$, satisfies the functional equation

$$
\Phi_{F}(s)=w \overline{\Phi_{F}(1-\bar{s})} .
$$

The numbers $\lambda_{j}$ and $\mu_{j}$ are called the spectral parameters of $F$.

The Selberg class of functions, introduced in [16], consists of functions $F \in \mathcal{S}^{\sharp}$ which satisfy the Ramanujan conjecture and are such that $\log F(s)=\sum_{n=1}^{\infty} b_{F}(n) n^{-s}$, where $b_{F}(n)$ are zero unless $n=p^{m}$ with $m \geqslant 1$ and a prime $p$ and $b_{F}(n) \ll n^{\theta}$ for some $\theta<1 / 2$.

It is conjectured that the Selberg class contains all $L$-functions possessing an Euler product with coefficients in the Dirichlet series suitably normalized so that they satisfy

Received 21 August 2015; revised 29 April 2016.

2010 Mathematics Subject Classification 11M26, 11M36 (primary), 11M41 (secondary).

This work was supported by the Simons Foundation (\#244988 to A.B.), the Academy of Finland (grant no. 138522 to A.-M. E.-H.), the Finnish Cultural Foundation (to A.-M. E.-H.) and the Federal Ministry of Education and Science (grant no. 05-39-3633-1/14 to A.O. and L.S.). 
the Ramanujan conjecture, that is, $L$-functions whose Dirichlet series representation has multiplicative coefficients $a(n)$ such that for any $\varepsilon>0$ we have $a(n) \ll n^{\varepsilon}$. Moreover, it is conjectured that the generalized Riemann hypothesis holds true for all functions $F \in \mathcal{S}$, meaning that all non-trivial zeros of $F$ are on the line $\operatorname{Re}(s)=1 / 2$. If a function from $\mathcal{S}^{\sharp}$ does not have an Euler product or does not satisfy the Ramanujan conjecture, it is not expected to satisfy the generalized Riemann hypothesis. There are numerous examples of functions that belong to $\mathcal{S}^{\sharp}$ and possess zeros off the critical line $\operatorname{Re}(s)=1 / 2$ and even off the critical strip $0 \leqslant \operatorname{Re}(s) \leqslant 1$. Therefore, the question of existence and location of zero-free regions remains an important open question for such functions.

A well-known example of a function in $\mathcal{S}^{\sharp}$ with zeros in the half-plane $\operatorname{Re}(s)>1$ is the Davenport-Heilbronn $L$-function $L_{\mathrm{DH}}(s)$ introduced in [5]. However, there exists $\sigma_{\mathrm{DH}}>1$ such that $L_{\mathrm{DH}}(s)$ is non-vanishing for all $s$ in the half-plane $\operatorname{Re}(s)>\sigma_{\mathrm{DH}}$. In [2], it is shown that the approximate value of the smallest such $\sigma_{\mathrm{DH}}$, up to six decimal places, is 1.120362 . Actually, Bombieri and Ghosh in [2] conducted an investigation of a certain Dirichlet series, called a series of Davenport-Heilbronn type, and, in the case when coefficients in the Dirichlet series are real or purely imaginary numbers, derived an equation satisfied by the smallest value $\sigma_{L}>1$ such that the Davenport-Heilbronn-type $L$-series $L(s)$ has no zeros in the half-plane $\operatorname{Re}(s)>\sigma_{L}$.

Another arithmetic example of a function from $\mathcal{S}^{\sharp}$ that does not possess an Euler product is the cubic $L$-function $L_{c}(s)$, defined and studied by Proskurin in [15], who proved that the function $L_{c}(s)$ possesses both zeros off the critical strip $0 \leqslant \operatorname{Re}(s) \leqslant 1$ and a zero-free region.

Therefore, for functions in $\mathcal{S}^{\sharp}$ that do not have an Euler product representation, it is of interest to define a criterion for zero-free regions. For functions in $\mathcal{S}^{\sharp}$ that possess an Euler product representation, Droll [6] formulated a very simple positivity criterion for zero-free regions stating that for $\tau \in[1,2]$, a function $F \in \mathcal{S}^{\text {\#b }}$ has no zeros in the half-plane $\operatorname{Re}(s)>\tau / 2$ (or, equivalently, no zeros in the half-plane $\operatorname{Re}(s)<1-\tau / 2)$ if and only if the sequence of real numbers $\operatorname{Re}\left(\lambda_{F}(n, \tau)\right)$, defined below, is non-negative for all $n \geqslant 1$.

Here, $\mathcal{S}^{\text {th }}$ is a certain subclass of the extended Selberg class introduced in [17] and $\lambda_{F}(n, \tau)$ denotes the $n$th $\tau$-Li coefficient associated to the function $F$ and defined for an integer $n$ and a real parameter $\tau \in[1,2]$ by

$$
\lambda_{F}(n, \tau)=\sum_{\rho \in Z(F)}^{*}\left(1-\left(\frac{\rho}{\rho-\tau}\right)^{n}\right),
$$

where $Z(F)$ stands for the set of non-trivial zeros of $F$ and $*$ indicates that the series converges in the $*$-sense, that is, as a $\operatorname{limit}_{T \rightarrow \infty} \sum_{\rho \in Z(F),|\operatorname{Im}(\rho)|<T}$.

For $F \in \mathcal{S}^{\sharp}$, it can be proved that the series (1.2) is convergent, by a method similar to the one presented in [11]. Namely, a straightforward application of the argument principle yields formulas for the distribution of zeros in the upper and lower half-planes, which imply that, for large positive $T$, the number of zeros $\rho \in Z(F)$ such that $0 \leqslant \operatorname{Im}(\rho) \leqslant T$ and the number of zeros $\rho \in Z(F)$ such that $0 \geqslant \operatorname{Im}(\rho) \geqslant-T$ differ at most by $O(\log T)$. Then the application of the method presented in [11] yields that the series $\sum_{\rho \in Z(F)}^{*}(1 / \rho)$ is convergent. Since $F$ is of order one, this is sufficient to conclude that the series (1.2) is convergent.

On the other hand, the functional equation axiom yields that non-trivial zeros of $F \in \mathcal{S}^{\sharp}$ are symmetric with respect to the critical line $\operatorname{Re}(s)=1 / 2$ and no further symmetry exists in general; hence, it is very difficult to derive a different expression for the series (1.2). Especially, it is hard to relate the series $\sum_{\rho \in Z(F)}^{*}(1 /(s-\rho))$ to the logarithmic derivative at $s \notin Z(F)$ of the complete function $\xi_{F}(s)$ defined below, a relation which is important in our method for fast computation of $\lambda_{F}(n, \tau)$. 
Therefore, in this paper we define $\tau$-Li coefficients for a subclass $\mathcal{S}_{\mathbb{R}}^{\sharp}$ of the extended Selberg class consisting of Dirichlet series (1.1) from $\mathcal{S}^{\sharp}$ with real coefficients $a_{F}(n)$ and real spectral parameters $\mu_{j}, j=1, \ldots, r$, in axiom (iii). A subclass $\mathcal{S}_{\mathbb{R}}^{\sharp}$ is chosen for two reasons: first, it arises naturally in many cases of number-theoretical interest and contains all arithmetic $L$-functions with real coefficients, including those with non-multiplicative coefficients in the Dirichlet series representation (such as cubic $L$-functions [15], Davenport-Heilbronn $L$-functions [5], Davenport-Heilbronn-type $L$-functions [2] etc); second, in this class, due to the reflection principle, zeros are symmetric with respect to the real line and hence it is possible to relate the series $\sum_{\rho \in Z(F)}^{*}(1 /(s-\rho))$ to $\xi_{F}^{\prime}(s) / \xi_{F}(s)$ for $s \notin Z(F)$.

We investigate analytically and numerically the behavior of the coefficients $\lambda_{F}(n, \tau)$ for large sets of positive integers $n$ and for different real values of $\tau$. Analytically, we prove the $\tau$-Li criterion for zero-free regions of functions in the class $\mathcal{S}_{\mathbb{R}}^{\sharp}$ and derive different formulas for the evaluation of the $\tau$-Li coefficients.

Then we focus on three examples of functions from $\mathcal{S}_{\mathbb{R}}^{\sharp} \backslash \mathcal{S}$ and investigate numerically the behavior of $\tau$-Li coefficients attached to those functions for different values of $\tau$. Numerical computations are based on the representation of $\tau$-Li coefficients of $F \in \mathcal{S}_{\mathbb{R}}^{\sharp}$ in terms of coefficients in the Taylor (Laurent) series representation of $F$ and conducted using the interval arithmetic package Arb, a C library for arbitrary-precision floating-point ball arithmetic, developed by Johansson [8]. We also discuss the precision of the numerical results.

\section{Properties of the class $\mathcal{S}_{\mathbb{R}}^{\sharp}$}

In this section we derive some basic analytic properties of the functions from $\mathcal{S}_{\mathbb{R}}^{\sharp}$. For $F \in \mathcal{S}_{\mathbb{R}}^{\sharp}$, we define the complete function by

$$
\xi_{F}(s)=s^{m_{F}}(s-1)^{m_{F}} \Phi_{F}(s) .
$$

The zeros of $\xi_{F}$ are called the non-trivial zeros of $F \in \mathcal{S}^{\sharp}$. The set of non-trivial zeros of $F$ is denoted by $Z(F)$. The properties of the class $\mathcal{S}_{\mathbb{R}}^{\sharp}$ are summarized in the following proposition.

Proposition 2.1. Let $F \in \mathcal{S}_{\mathbb{R}}^{\sharp}$. The following statements are true.

(i) $Z(F)=1-Z(F)$.

(ii) The function $F$ is a meromorphic function of order one, with at most one pole. If it exists, the pole is situated at $s=1$.

(iii) The complete function $\xi_{F}$ satisfies the functional equation $\xi_{F}(s)= \pm \xi_{F}(1-s)$.

(iv) There exists a number $\sigma_{0} \geqslant 1$ such that $\xi_{F}(s) \neq 0$ for all $s$ with $\operatorname{Re}(s)>\sigma_{0}$.

(v) Assume additionally that $0 \notin Z(F)$. Then the series $\sum_{\rho \in Z(F)}(s-\rho)^{-1}$ is $*$-convergent for all $s \in \mathbb{C} \backslash Z(F)$ and one has

$$
\frac{\xi_{F}^{\prime}}{\xi_{F}}(s)=\sum_{\rho \in Z(F)}^{*} \frac{1}{s-\rho} .
$$

Proof. (i) The statement follows from the functional equation in axiom (iii) and the reflection principle.

(ii) The statement is a special case of [17, Lemma 3.3].

(iii) The coefficients $a_{F}(n)$ of the Dirichlet series (1.1) are real and hence $\overline{F(\bar{s})}=F(s)$. Furthermore, for $j=1, \ldots, r$, we have $\lambda_{j}, \mu_{j} \in \mathbb{R}$ and hence $\overline{\xi_{F}(\bar{s})}=\xi_{F}(s)$. Therefore, the functional equation from axiom (iii) reads as $\xi_{F}(s)=w \xi_{F}(1-s)$ for some complex constant $w$ of modulus one. Since the function $\xi_{F}(s)$ attains real values for all real $s$, we deduce $w= \pm 1$. 
(iv) Let $K$ denote the smallest positive integer such that $a_{F}(K) \neq 0$. Then we have

$$
|F(s)| \geqslant \frac{\left|a_{F}(K)\right|}{K^{\operatorname{Re}(s)}}-\sum_{n=K+1}^{\infty} \frac{\left|a_{F}(n)\right|}{n^{\operatorname{Re}(s)}} .
$$

For $\operatorname{Re}(s) \geqslant \alpha>1$ and $n \geqslant K+1$, it is obvious that

$$
\frac{\left|a_{F}(n)\right|}{(n / K)^{\operatorname{Re}(s)}} \leqslant \frac{\left|a_{F}(n)\right|}{(n / K)^{\alpha}}=K^{\alpha} \frac{\left|a_{F}(n)\right|}{(n)^{\alpha}}
$$

and hence the absolute convergence of the Dirichlet series (1.1) for $\operatorname{Re}(s)>1$ implies that the series $\sum_{n=K+1}^{\infty}\left|a_{F}(n)\right| /(n / K)^{\operatorname{Re}(s)}$ converges uniformly in the half-plane $\operatorname{Re}(s) \geqslant \alpha>1$. Therefore,

$$
\lim _{\operatorname{Re}(s) \rightarrow+\infty} \sum_{n=K+1}^{\infty} \frac{\left|a_{F}(n)\right|}{(n / K)^{\operatorname{Re}(s)}}=\sum_{n=K+1}^{\infty} \lim _{\operatorname{Re}(s) \rightarrow+\infty} \frac{\left|a_{F}(n)\right|}{(n / K)^{\operatorname{Re}(s)}}=0
$$

and hence there exists $\sigma_{0} \geqslant 1$ such that for every $s$ with $\operatorname{Re}(s)>\sigma_{0}$, we have

$$
\left|a_{F}(K)\right|>\sum_{n=K+1}^{\infty} \frac{\left|a_{F}(n)\right|}{(n / K)^{\operatorname{Re}(s)}}
$$

or, equivalently,

$$
\frac{\left|a_{F}(K)\right|}{K^{\operatorname{Re}(s)}}-\sum_{n=K+1}^{\infty} \frac{\left|a_{F}(n)\right|}{n^{\operatorname{Re}(s)}}>0,
$$

which yields that $|F(s)| \neq 0$ for all $s$ in the half-plane $\operatorname{Re}(s)>\sigma_{0}$. Since the gamma function has no zeros, this yields the statement.

(v) Since $Z(F)=1-Z(F)$, the non-trivial zeros of $F \in \mathcal{S}_{\mathbb{R}}^{\sharp}$ may be paired as $\rho=\sigma+i$ and $1-\rho=1-\sigma-i t$. Moreover, $0 \notin Z(F)$ implies $1 \notin Z(F)$ and hence we have

$$
\sum_{\substack{\rho \in Z(F),|\operatorname{Im} \rho| \leqslant T}} \frac{1}{\rho}=\sum_{\rho \in Z(F) \cap \mathbb{R}} \frac{1}{\rho}+\sum_{\substack{\rho \in Z(F) \\ 0<\operatorname{Im} \rho \leqslant T}}\left(\frac{1}{\rho}+\frac{1}{1-\rho}\right)=\sum_{\rho \in Z(F) \cap \mathbb{R}} \frac{1}{\rho}+\sum_{\substack{\rho \in Z(F) \\ 0<\operatorname{Im} \rho \leqslant T}} \frac{1}{\rho(1-\rho)} .
$$

Part (iv) together with the functional equation yields that $Z(F) \subseteq\left\{z \in \mathbb{C}: 1-\sigma_{0} \leqslant\right.$ $\left.\operatorname{Re}(s) \leqslant \sigma_{0}\right\}$. Zeros of entire functions form a discrete subset of $\mathbb{C}$ and hence the first sum in the above equation is finite. Since $\xi_{F}$ is an order-one entire function, the second sum in the above equation converges as $T \rightarrow+\infty$; therefore, the series $\sum_{\rho \in Z(F)}(1 / \rho)$ is $*$-convergent and

$$
\sum_{\rho \in Z(F)}^{*} \frac{1}{\rho}=\sum_{\rho \in Z(F)}^{*} \frac{1}{1-\rho} .
$$

When $0 \notin Z(F)$, the Hadamard factorization theorem and (ii) imply that the function $\xi_{F}$ can be represented as a product over its zeros

$$
\xi_{F}(s)=\xi_{F}(0) e^{b_{F} s} \prod_{\rho \in Z(F)}\left(1-\frac{s}{\rho}\right) e^{s / \rho},
$$

where $b_{F}=\xi_{F}^{\prime}(0) / \xi_{F}(0)$. Therefore,

$$
\frac{\xi_{F}^{\prime}}{\xi_{F}}(s)=b_{F}+\sum_{\rho \in Z(F)}\left(\frac{1}{s-\rho}+\frac{1}{\rho}\right)
$$


for all $s \in \mathbb{C} \backslash Z(F)$. Specializing to $s=1$, we obtain

$$
\frac{\xi_{F}^{\prime}}{\xi_{F}}(1)=b_{F}+\sum_{\rho \in Z(F)}\left(\frac{1}{1-\rho}+\frac{1}{\rho}\right)=b_{F}+2 \sum_{\rho \in Z(F)}^{*} \frac{1}{\rho} .
$$

The statement (iii) implies that

$$
0=\frac{\xi_{F}^{\prime}}{\xi_{F}}(1)+\frac{\xi_{F}^{\prime}}{\xi_{F}}(0)=2\left(b_{F}+\sum_{\rho \in Z(F)}^{*} \frac{1}{\rho}\right)
$$

and hence

$$
b_{F}=\frac{\xi_{F}^{\prime}}{\xi_{F}}(0)=-\sum_{\rho \in Z(F)}^{*} \frac{1}{\rho} .
$$

This, together with formula (2.2), completes the proof.

3. Li-type criterion for zero-free regions of functions in the class $\mathcal{S}_{\mathbb{R}}^{\sharp}$

In this section we prove that the $\tau$-Li coefficients attached to a function $F \in \mathcal{S}_{\mathbb{R}}^{\sharp}$ are well defined, derive three equivalent formulas for computing them and prove the $\tau$-Li criterion for zero-free regions of $F$.

Proposition 3.1. Fix $\tau \in[1,+\infty)$, an arbitrary real number. Let $F \in \mathcal{S}_{\mathbb{R}}^{\sharp}$ with the property that $0, \tau \notin Z(F)$. Then the sum (1.2) defining $\lambda_{F}(n, \tau)$ is $*$-convergent for every positive integer $n$.

Proof. By Proposition 2.1(v), the series $\sum_{\rho \in Z(F)} 1 / \rho$ is $*$-convergent and so is the series $\sum_{\rho \in Z(F)} \tau / \rho$.

Let $Z_{\tau}(F)=\{\rho / \tau: \rho \in Z(F)\}$. Then $Z(F)=1-Z(F)$ implies $Z_{\tau}(F)=\{(1-\rho) / \tau: \rho \in$ $Z(F)\}$.

Since $\xi_{F}$ is an entire function of order one, the series $\sum_{\rho \in Z(F)} 1 /(1-\rho)^{k}$ converges absolutely for every integer $k \geqslant 2$ and so does the series $\sum_{\rho \in Z_{\tau}(F)} 1 /(1-\rho)^{k}$. Now, we have

$$
\lambda_{F}(n, \tau)=\sum_{\rho \in Z_{\tau}(F)}^{*}\left(1-\left(\frac{\rho}{\rho-1}\right)^{n}\right)=n \sum_{\rho \in Z_{\tau}(F)}^{*} \frac{1}{1-\rho}+\sum_{k=2}^{n}\left(\begin{array}{l}
n \\
k
\end{array}\right) \sum_{\rho \in Z_{\tau}(F)}^{*} \frac{(-1)^{k-1}}{(1-\rho)^{k}}
$$

and both series on the right-hand side are convergent. The proof is complete.

Remark 1. For $F \in \mathcal{S}_{\mathbb{R}}^{\sharp}$ and $\tau \in[1, \infty)$, the coefficients $\lambda_{F}(n, \tau)$ are real.

Indeed, by the reflection principle, $Z(F)=\overline{Z(F)}$ and hence $(1.2)$ implies $\lambda_{F}(n, \tau) \in \mathbb{R}$.

Let $d_{F}\left(n, z_{0}\right)$ be the power series coefficients in the expansion of the logarithmic derivative of $\xi_{F}(1 /(1-s))$ around the point $z_{0} \neq 1$, which is not a zero of $\xi_{F}(1 /(1-s))$, that is, assume that in a small neighborhood of $z_{0}$, we have

$$
\frac{d}{d s} \log \xi_{F}\left(\frac{1}{1-s}\right)=\sum_{n=0}^{\infty} d_{F}\left(n, z_{0}\right)\left(s-z_{0}\right)^{n}
$$

Performing calculations analogous to those in the proofs of [6, Lemma 2.1.2] and [7, Theorem $15]$ and keeping in mind the properties of the class $\mathcal{S}_{\mathbb{R}}^{\sharp}$, we are able to derive alternate definitions of the $\tau$-Li coefficients attached to $F \in \mathcal{S}_{\mathbb{R}}^{\sharp}$. 
Proposition 3.2. Let $F \in \mathcal{S}_{\mathbb{R}}^{\sharp}$ and let $\tau \in[1, \infty)$ be an arbitrary fixed real number such that $0, \tau \notin Z(F)$. For every positive integer $n$, one has

$$
\lambda_{F}(n, \tau)=\frac{\tau}{(n-1) !}\left[\frac{d^{n}}{d s^{n}}\left(s^{n-1} \log \xi_{F}(s)\right)\right]_{s=\tau}=\frac{1}{\tau^{n}} d_{F}\left(n-1,1-\frac{1}{\tau}\right) .
$$

Proof. The proof of (3.2) follows the lines of the proof of [6, Lemma 2.1.2], which may be applied since the function $\xi_{F}$ is an entire function of order one that satisfies (2.1).

The following theorem is the Li-type criterion for the zero-free regions of functions from $\mathcal{S}_{\mathbb{R}}^{\sharp}$.

Theorem 3.3. Let $F \in \mathcal{S}_{\mathbb{R}}^{\sharp}$ and let $\tau \in[1, \infty)$ be such that $0, \tau \notin Z(F)$. Then the following statements are equivalent.

(i) $\xi_{F}$ possesses no zeros in the half-plane $\operatorname{Re}(s)>\tau / 2$.

(ii) $\lambda_{F}(n, \tau) \geqslant 0$ for all $n \geqslant 1$.

(iii) For every fixed $\delta>0$, there exists a constant $c(\delta)$ such that $\lambda_{F}(n, \tau) \geqslant-c(\delta) \exp (\delta n)$.

(iv)

$$
\limsup _{n \rightarrow \infty}\left|\lambda_{F}(n+1, \tau)\right|^{1 / n} \leqslant 1 .
$$

Proof. It is obvious that (ii) implies (iii). First, we prove that (i) is equivalent to (iv). Then we prove that (i) implies (ii) and that (iii) implies (i).

(i) $\Leftrightarrow$ (iv) The assumption $\tau \notin Z(F)$ implies that the point $\tau_{0}=1-1 / \tau$ is not a zero of the function $\xi_{F}(1 /(1-s))$; hence, by $(3.2)$, in a small neighborhood of $\tau_{0}$, one has

$$
\frac{d}{d s} \log \xi_{F}\left(\frac{1}{1-s}\right)=\sum_{n=0}^{\infty} \tau^{n+1} \lambda_{F}(n+1, \tau)\left(s-\tau_{0}\right)^{n} .
$$

The conformal mapping $w=1 /(1-s)$ maps the disc $D(1-1 / \tau, 1 / \tau)=\{z:|z-1+1 / \tau|<1 / \tau\}$ onto the half-plane $\operatorname{Re}(w)>\tau / 2$. Therefore, the function $\xi_{F}(w)=\xi_{F}(1 /(1-s))$ has no zeros in the half-plane $\operatorname{Re}(w)>\tau / 2$ if and only if the function $\log \xi_{F}(1 /(1-s))$ or, equivalently, the function $(d / d s) \log \xi_{F}(1 /(1-s))$ is holomorphic inside $D(1-1 / \tau, 1 / \tau)$. This is equivalent to the statement that the radius of convergence of the power series (3.4) is greater than or equal to $1 / \tau$. Therefore, the function $\xi_{F}(w)$ has no zeros in the half-plane $\operatorname{Re}(w)>\tau / 2$ if and only if

$$
\limsup _{n \rightarrow \infty}\left|\tau^{n+1} \lambda_{F}(n+1, \tau)\right|^{1 / n} \leqslant \tau,
$$

a condition equivalent to (3.3).

(i) $\Rightarrow$ (ii) A straightforward computation shows that

$$
\left|\frac{\rho}{\rho-\tau}\right|^{2}=1+2 \tau \frac{\operatorname{Re}(\rho)-\tau / 2}{|\rho-\tau|^{2}} .
$$

Since $\operatorname{Re}(\rho) \leqslant \tau / 2$ for all $\rho \in Z(F)$, (3.5) implies $|\rho /(\rho-\tau)| \leqslant 1$ for all $\rho \in Z(F)$. Therefore, $\lambda_{F}(n, \tau) \geqslant 0$ for all positive integers $n$.

(iii) $\Rightarrow$ (i) Assume that (i) does not hold true, that is, assume that there exists $\rho \in Z(F)$ such that $\operatorname{Re}(\rho)>\tau / 2$. Then equation (3.5) implies that, for such $\rho$, one has $|\rho /(\rho-\tau)|>1$. Since $\tau$ is fixed, there exists a maximal value of $|\rho /(\rho-\tau)|=|1+\tau /(\rho-\tau)|$ that is attained at the finite subset $Z_{\max }(F)=\left\{\rho_{1}, \ldots, \rho_{M}\right\}$ of elements of $Z(F)$ with real part greater than $\tau / 2$. Let us put

$$
\max _{\rho \in Z(F)}\left\{\left|\frac{\rho}{\rho-\tau}\right|\right\}=1+m>1
$$


Since $|\rho /(\rho-\tau)|=|\bar{\rho} /(\bar{\rho}-\tau)|$ and $Z(F)=\overline{Z(F)}$, it is obvious that elements of $Z_{\max }(F)$ are either real or come in conjugate pairs. We put $M=2 k+l$, where $k$ is the number of complexconjugate pairs of elements of $Z_{\max }(F), l \geqslant 0$ is the number of real elements of $Z_{\max }(F)$ and we numerate the set $Z_{\max }(F)$ so that $\rho_{j+k}=\overline{\rho_{j}}$ for $j=1, \ldots, k$ and the real zeros are $\rho_{2 k+i}$, $i=0, \ldots, l$. Note that if $1 / 2 \in Z(F)$, we have, as $\tau \geqslant 1$,

$$
\left|\frac{1 / 2}{1 / 2-\tau}\right|=\frac{1}{2 \tau-1} \leqslant 1
$$

so $1 / 2 \notin Z_{\max }(F)$. Moreover, one cannot have both $\rho \in Z_{\max }(F)$ and $(1-\rho) \in Z_{\max }(F)$, since one of them has real part which is less than or equal to $\tau / 2$.

Furthermore, since zeros of an entire function do not accumulate around any finite point, there exists $\epsilon>0$ such that

$$
\left|\frac{\rho}{\rho-\tau}\right| \leqslant 1+m-\epsilon \quad \text { for all } \rho \in Z(F) \backslash Z_{\max }(F) .
$$

Assume $1 / 2 \notin Z(F)$. Using the fact that $Z(F)=1-Z(F)$, we may write $\lambda_{F}(n, \tau)$ as

$$
\begin{aligned}
& \lambda_{F}(n, \tau)=\sum_{j=1}^{k}\left(2-2 \operatorname{Re}\left(\frac{\rho_{j}}{\rho_{j}-\tau}\right)^{n}\right)+\sum_{j=1}^{k}\left(2-2 \operatorname{Re}\left(\frac{1-\rho_{j}}{1-\rho_{j}-\tau}\right)^{n}\right) \\
& +\sum_{j=2 k+1}^{2 k+l}\left(2-\left(\frac{\rho_{j}}{\rho_{j}-\tau}\right)^{n}-\left(\frac{1-\rho_{j}}{1-\rho_{j}-\tau}\right)^{n}\right) \\
& +\frac{1}{2} \sum_{\substack{\rho \in Z(F) \\
\rho \notin Z_{\max }(F), 1-\rho \notin Z_{\max }(F)}}\left(2-\left(\frac{\rho}{\rho-\tau}\right)^{n}-\left(\frac{1-\rho}{1-\rho-\tau}\right)^{n}\right),
\end{aligned}
$$

with the convention that in the case when $k=0$ or $l=0$ the empty sum is equal to zero.

Let $\phi_{j}$ denote the argument of $\rho_{j} /\left(\rho_{j}-\tau\right), j=1, \ldots, k$; then the first sum on the right-hand side of (3.7) is equal to

$$
2 k-2(1+m)^{n} \sum_{j=1}^{k} \cos \left(n \phi_{j}\right) .
$$

The inequality $\operatorname{Re}\left(\rho_{j}\right)>\tau / 2$ implies $1-\operatorname{Re}\left(\rho_{j}\right)-\tau / 2<0$; hence, applying formula (3.5) to $\rho=1-\rho_{j}$ yields that $\left|\left(1-\rho_{j}\right) /\left(1-\rho_{j}-\tau\right)\right|<1$. Therefore, the second sum on the right-hand side of (3.7) is dominated by $4 k$.

For real $\rho_{j} \in Z_{\max }(F)$, it is obvious that $\left(\rho_{j} /\left(\rho_{j}-\tau\right)\right)^{n}=( \pm 1)^{n}(1+m)^{n}$, where the sign is equal to the sign of the difference $\rho_{j}-\tau$. In this case, $\left|\left(1-\rho_{j}\right) /\left(1-\rho_{j}-\tau\right)\right|<1$; hence, the sum in the second line of formula (3.7) is $-l_{1}(1+m)^{n}-l_{2}(-1)^{n}(1+m)^{n}+O(4 l)$, where $l_{1}$ is the number of indices $j \in\{2 k+1, \ldots, 2 k+l\}$ such that $\rho_{j}>\tau$ and $l_{2}=l-l_{1}$.

It is left to estimate the sum in the third line of (3.7). In order to do so, we write $\left\{\rho \in Z(F): \rho \notin Z_{\max }(F), 1-\rho \notin Z_{\max }(F)\right\}$ as a disjoint union $Z_{1}(F) \uplus Z_{2}(F)$, where $Z_{1}(F)=\left\{\rho \in Z(F): \rho \notin Z_{\max }(F), 1-\rho \notin Z_{\max }(F)\right.$ and $\left.\min \{|\rho|,|1-\rho|\}>n \tau\right\}$. For $\rho \in Z_{1}(F)$, one has $n|\tau / \rho|<1$; hence, $n^{k}|\tau / \rho|^{k}<n^{2}|\tau / \rho|^{2}$ for all integers $k \geqslant 3$. Therefore, for $\rho \in Z_{1}(F)$, we have

$$
\left(\frac{\rho}{\rho-\tau}\right)^{n}=\left(1-\frac{\tau}{\rho}\right)^{-n}=1+\frac{n \tau}{\rho}+O\left(n^{2}\left|\frac{\tau}{\rho}\right|^{2}\right) \quad \text { as }|\rho| \rightarrow \infty .
$$

Analogously, for $\rho \in Z_{1}(F)$, one gets

$$
\left(\frac{1-\rho}{1-\rho-\tau}\right)^{n}=1+\frac{n \tau}{1-\rho}+O\left(n^{2}\left|\frac{\tau}{1-\rho}\right|^{2}\right) \quad \text { as }|\rho| \rightarrow \infty
$$


Therefore,

$$
\begin{aligned}
& \sum_{\rho \in Z_{1}(F)}\left(2-\left(\frac{\rho}{\rho-\tau}\right)^{n}-\left(\frac{1-\rho}{1-\rho-\tau}\right)^{n}\right) \\
& =-n \tau \sum_{\rho \in Z_{1}(F)} \frac{1}{\rho(1-\rho)}+O\left(2 n^{2} \tau^{2} \sum_{\rho \in Z(F)} \frac{1}{|\rho|^{2}}\right)=O\left(n^{2} \tau^{2}\right)
\end{aligned}
$$

since the function $\xi_{F}$ has order one, so both series on the right-hand side of (3.8) are convergent.

It is left to estimate the sum over $Z_{2}(F)$. Since the series $\sum_{\rho \in Z(F)}|\rho|^{-\mu}$ and $\sum_{\rho \in Z(F)} \mid \rho-$ $\left.\tau\right|^{-\mu}$ converge for all real numbers $\mu>1$, the number of elements of the set $Z_{2}(F)$ is bounded by $C n^{2}$ for some positive constant $C$ depending upon $F$ and $\tau$; hence, the bound (3.6) implies

$$
\frac{1}{2} \sum_{\rho \in Z_{2}(F)}\left(2-\left(\frac{\rho}{\rho-\tau}\right)^{n}-\left(\frac{1-\rho}{1-\rho-\tau}\right)^{n}\right)=O\left(n^{2}(1+m-\epsilon)^{n}\right) .
$$

Inserting this, together with (3.8), into (3.7), we get, for even values of $n$,

$$
\lambda_{F}(n, \tau)=2 k-2(1+m)^{n} \sum_{j=1}^{k} \cos \left(n \phi_{j}\right)-l(1+m)^{n}+O\left(n^{2} \tau^{2}\right)+O\left(n^{2}(1+m-\epsilon)^{n}\right) .
$$

Note that, if $1 / 2$ is a zero, then $1 / 2 \in Z_{2}(F)$ and it is taken into account in the above estimate.

Applying Dirichlet's theorem on simultaneous Diophantine approximations, we can make the $\operatorname{sum} \sum_{j=1}^{k} \cos \left(n \phi_{j}\right)$ arbitrarily close to $k$. Since $k$ and $l$ cannot both be equal to zero, this shows that the value of $\lambda_{F}(n, \tau)$ can be infinitely many times negative and exponentially large in amplitude, which contradicts (iii).

Using the functional equation from axiom (iii), we immediately deduce the following consequence of Theorem 3.3.

Corollary 3.4. Let $F \in \mathcal{S}_{\mathbb{R}}^{\sharp}$ and let $\tau \in[1, \infty)$ be such that $0, \tau \notin Z(F)$. The following statements are equivalent.

(i) All the non-trivial zeros of the function $F$ lie in the strip $1-\tau / 2 \leqslant \operatorname{Re}(s) \leqslant \tau / 2$.

(ii) The $\tau$-Li coefficients $\lambda_{F}(n, \tau) \geqslant 0$ for all $n \geqslant 1$.

(iii) For every fixed $\delta>0$, there exists a constant $c(\delta)$ such that $\lambda_{F}(n, \tau) \geqslant-c(\delta) \exp (\delta n)$.

(iv) The $\tau$-Li coefficients of $F$ have the property that

$$
\limsup _{n \rightarrow \infty}\left|\lambda_{F}(n+1, \tau)\right|^{1 / n} \leqslant 1
$$

\section{Some examples of functions in $\mathcal{S}_{\mathbb{R}}^{\sharp}$}

In [2], Bombieri and Ghosh considered the twisted Dirichlet series of Davenport-Heilbronn type defined by

$$
f(s, \xi ; \psi)=\sum_{n=1}^{\infty} \frac{a(n, \xi) \psi(n)}{n^{s}},
$$

where $\xi$ is a complex number, $\psi$ is a completely multiplicative function satisfying the condition $|\psi(p)|<p$ for all primes $p$ and the coefficients $a(n, \xi)$ are defined as 


$$
a(n, \xi)= \begin{cases}1, & n \equiv 1(\bmod 5) \\ \xi, & n \equiv 2(\bmod 5) \\ -\xi, & n \equiv 3(\bmod 5) \\ -1, & n \equiv 4(\bmod 5) \\ 0, & n \equiv 0(\bmod 5)\end{cases}
$$

In this section we consider in greater detail two special cases of the function $f(s, \xi ; \psi)$. We also define a new Dirichlet series of similar type using congruence modulo 7 and two parameters $\xi$ and $\rho$. We deduce sufficient conditions for those functions to belong to the class $\mathcal{S}^{\sharp}$ and, further, to $\mathcal{S}_{\mathbb{R}}^{\sharp}$.

EXAMPLE 1 . When $\psi \equiv 1$ and $\xi=\xi_{+}=-\phi+\sqrt{1+\phi^{2}}$, where $\phi=(1+\sqrt{5}) / 2$ is the golden ratio, the Dirichlet series $f(s, \xi ; \psi)$ coincides with the Davenport-Heilbronn $L$-function; see for example $[\mathbf{1 8}, \S 10.25]$. Actually, $\xi_{+}$is one solution of the quadratic equation

$$
\sin \left(\frac{4 \pi}{5}\right)+\xi \sin \left(\frac{8 \pi}{5}\right)=\xi\left(\sin \left(\frac{2 \pi}{5}\right)+\xi \sin \left(\frac{4 \pi}{5}\right)\right)
$$

whose second solution is $\xi_{-}=-\phi-\sqrt{1+\phi^{2}}$.

Let us put $L_{\mathrm{DH}}(s, \xi)=f(s, \xi ; \psi)$, where $f(s, \xi ; \psi)$ is the series defined by (4.1) with $\psi \equiv 1$ and $\xi$ is a complex number. Then, following $[\mathbf{1 8}, \S 10.25]$, we easily see that the function $L_{\mathrm{DH}}\left(s, \xi_{ \pm}\right)$satisfies the functional equation

$$
\left(\frac{5}{\pi}\right)^{s / 2} \Gamma\left(\frac{s+1}{2}\right) L_{\mathrm{DH}}\left(s, \xi_{ \pm}\right)= \pm\left(\frac{5}{\pi}\right)^{(1-s) / 2} \Gamma\left(1-\frac{s}{2}\right) L_{\mathrm{DH}}\left(1-s, \xi_{ \pm}\right)
$$

Actually, we can say more about the function $L_{\mathrm{DH}}(s, \xi)$ when the parameter $\xi$ takes real values.

Proposition 4.1. Assume that the function $L_{\mathrm{DH}}(s, \xi)$ with real $\xi$ has a zero outside the critical strip $0 \leqslant \operatorname{Re}(s) \leqslant 1$ (or outside the critical line under the generalized Riemann hypothesis for the $L$-functions $L(s, \chi)$, where $\chi$ is a character modulo 5$)$.

Then $L_{\mathrm{DH}}(s, \xi) \in \mathcal{S}_{\mathbb{R}}^{\sharp}$ if and only if $\xi=\xi_{ \pm}$, that is, $\xi$ is one of the solutions of the equation (4.2).

Proof. If $\xi=\xi_{ \pm}$are the solutions of $(4.2)$, it is obvious that $L_{\mathrm{DH}}\left(s, \xi_{ \pm}\right) \in \mathcal{S}_{\mathbb{R}}^{\sharp}$. Furthermore, in $[18, \S 10.25]$, it is proved that $L_{\mathrm{DH}}\left(s, \xi_{+}\right)$has a zero outside the critical strip $0 \leqslant \operatorname{Re}(s) \leqslant 1$. Bombieri and Ghosh [2] have proved that the function $L_{\mathrm{DH}}\left(s, \xi_{-}\right)$also possesses zeros outside the critical strip $0 \leqslant \operatorname{Re}(s) \leqslant 1$. Therefore, it is left only to show the converse statement.

Notice first that $L_{\mathrm{DH}}(s, \xi)$ has real coefficients and hence $L_{\mathrm{DH}}(s, \xi)=\overline{L_{\mathrm{DH}}(\bar{s}, \xi)}$. Therefore, the functional equation condition implies that if $L_{\mathrm{DH}}(s, \xi)=0$, then also $L_{\mathrm{DH}}(1-s, \xi)=0$. We may write

$$
L_{\mathrm{DH}}(s, \xi)=\frac{1-\xi i}{2} L(s, \chi)+\frac{1+\xi i}{2} L(s, \bar{\chi}),
$$

where $\chi$ is the character modulo 5 that takes the values $\chi(1)=1, \chi(2)=i, \chi(3)=-i, \chi(4)=$ -1 . Also,

$$
L_{\mathrm{DH}}(1-s, \xi)=\frac{1-\xi i}{2} L(1-s, \chi)+\frac{1+\xi i}{2} L(1-s, \bar{\chi}) .
$$

Hence, if $L_{\mathrm{DH}}(1-s, \xi)=0$, then

$$
\frac{1-\xi i}{2} L(1-s, \chi)+\frac{1+\xi i}{2} L(1-s, \bar{\chi})=0
$$


and thus

$$
L(1-s, \bar{\chi})=-\frac{1-\xi i}{1+\xi i} L(1-s, \chi) .
$$

On the other hand, if $L_{\mathrm{DH}}(s, \xi)=0$, then by the functional equation for the $L$-functions $L(s, \chi)$ and $L(s, \bar{\chi})$, we have

$$
\tau(\chi) \frac{1-\xi i}{2} L(1-s, \bar{\chi})+\tau(\bar{\chi}) \frac{1+\xi i}{2} L(1-s, \chi)=0 .
$$

Substituting (4.4), we obtain

$$
\tau(\chi) \frac{1-\xi i}{2}\left(-\frac{1-\xi i}{1+\xi i} L(1-s, \chi)\right)+\tau(\bar{\chi}) \frac{1+\xi i}{2} L(1-s, \chi)=0 .
$$

The above equation holds true for all zeros $s$ of $L_{\mathrm{DH}}(s, \xi)$. Now, we take $s$ to be the zero of $L_{\mathrm{DH}}(s, \xi)$ outside the critical strip $0 \leqslant \operatorname{Re}(s) \leqslant 1$ (or outside the critical line under the generalized Riemann hypothesis for the $L$-functions $L(s, \chi))$; hence, $L(1-s, \chi) \neq 0$ and the above equality becomes

$$
-\tau(\chi) \frac{(1-\xi i)^{2}}{1+\xi i}+\tau(\bar{\chi})(1+\xi i)=0
$$

Since

$$
\tau(\chi)=2 i \sin \left(\frac{2 \pi}{5}\right)-2 \sin \left(\frac{4 \pi}{5}\right)
$$

and

$$
\tau(\bar{\chi})=2 i \sin \left(\frac{2 \pi}{5}\right)+2 \sin \left(\frac{4 \pi}{5}\right)
$$

a simple computation shows that equation (4.2) is equivalent to (4.5). This completes the proof.

Example 2. Define the function

$$
L_{7}(s, \xi, \rho)=\sum_{m=1}^{\infty} \frac{b(m, \xi, \rho)}{m^{s}},
$$

where

$$
b(m, \xi, \rho)= \begin{cases}1, & m \equiv 1(\bmod 7) \\ \xi, & m \equiv 2(\bmod 7) \\ \rho, & m \equiv 3(\bmod 7) \\ -\rho, & m \equiv 4(\bmod 7) \\ -\xi, & m \equiv 5(\bmod 7) \\ -1, & m \equiv 6(\bmod 7) \\ 0, & m \equiv 0(\bmod 7)\end{cases}
$$

and the complex numbers $\xi$ and $\rho$ satisfy the system of equations

$$
\left\{\begin{array}{l}
\sin \left(\frac{4 \pi}{7}\right)+\xi \sin \left(\frac{8 \pi}{7}\right)+\rho \sin \left(\frac{12 \pi}{7}\right)=\bar{\xi}\left(\sin \left(\frac{2 \pi}{7}\right)+\xi \sin \left(\frac{4 \pi}{7}\right)+\rho \sin \left(\frac{6 \pi}{7}\right)\right) \\
\sin \left(\frac{6 \pi}{7}\right)+\xi \sin \left(\frac{12 \pi}{7}\right)+\rho \sin \left(\frac{18 \pi}{7}\right)=\bar{\rho}\left(\sin \left(\frac{2 \pi}{7}\right)+\xi \sin \left(\frac{4 \pi}{7}\right)+\rho \sin \left(\frac{6 \pi}{7}\right)\right) .
\end{array}\right.
$$


The properties of the function $L_{7}(s, \xi, \rho)$ can be summarized in the following proposition.

Proposition 4.2. For all complex values of $\xi$ and $\rho$ satisfying the system (4.7), the function $L_{7}(s, \xi, \rho)$ defined by (4.6) belongs to the class $\mathcal{S}^{\sharp}$ and satisfies the functional equation

$$
\left(\frac{7}{\pi}\right)^{s / 2} \Gamma\left(\frac{s+1}{2}\right) L_{7}(s, \xi, \rho)=\frac{2}{\sqrt{7}} \Xi(7)\left(\frac{7}{\pi}\right)^{(1-s) / 2} \Gamma\left(1-\frac{s}{2}\right) \overline{L_{7}(1-\bar{s}, \xi, \rho)},
$$

where

$$
\Xi(7):=\sin \left(\frac{2 \pi}{7}\right)+\xi \sin \left(\frac{4 \pi}{7}\right)+\rho \sin \left(\frac{6 \pi}{7}\right)
$$

Proof. First, we prove that if $\xi$ and $\rho$ are solutions of the system (4.7), then

$$
|\Xi(7)|^{2}=\frac{7}{4}
$$

Adding the trivial equation

$$
\sin \left(\frac{2 \pi}{7}\right)+\xi \sin \left(\frac{4 \pi}{7}\right)+\rho \sin \left(\frac{6 \pi}{7}\right)=\sin \left(\frac{2 \pi}{7}\right)+\xi \sin \left(\frac{4 \pi}{7}\right)+\rho \sin \left(\frac{6 \pi}{7}\right)
$$

as the first equation of the system (4.7), multiplying the $j$ th equation by $\sin \left(\frac{2 \pi j}{7}\right), j=1,2,3$, and taking the sum, we get the equation

$$
\sum_{j=1}^{3} \sin ^{2}\left(\frac{2 \pi j}{7}\right)+\xi \sum_{j=1}^{3} \sin \left(\frac{2 \pi j}{7}\right) \sin \left(\frac{4 \pi j}{7}\right)+\rho \sum_{j=1}^{3} \sin \left(\frac{2 \pi j}{7}\right) \sin \left(\frac{6 \pi j}{7}\right)=|\Xi(7)|^{2} .
$$

A simple application of trigonometric identities implies that the factors multiplying $\xi$ and $\rho$ in the above equation are equal to zero, while the constant term on the left-hand side is equal to $7 / 4$; hence, $|\Xi(7)|^{2}=7 / 4$. This shows that the term

$$
\frac{2}{\sqrt{7}} \Xi(7)
$$

that multiplies the right-hand side of the equation (4.8) is a complex number of modulus one.

The function given by (4.6) is a Dirichlet series with bounded coefficients and hence it converges absolutely in the half-plane $\operatorname{Re}(s)>1$. It is holomorphic also at $s=1$. Therefore, it is obvious that $L_{7}(s, \xi, \rho)$ satisfies the first two axioms of the class $\mathcal{S}^{\sharp}$. In order to prove that it belongs to $\mathcal{S}^{\sharp}$, it is left to prove the functional equation axiom. For that purpose we will write $L_{7}(s, \xi, \rho)$ as a linear combination of Hurwitz zeta functions $\zeta(s, z)$, namely,

$$
L_{7}(s, \xi, \rho)=\frac{1}{7^{s}}\left(\zeta\left(s, \frac{1}{7}\right)+\xi \zeta\left(s, \frac{2}{7}\right)+\rho \zeta\left(s, \frac{3}{7}\right)\right)-\frac{1}{7^{s}}\left(\rho \zeta\left(s, \frac{4}{7}\right)+\xi \zeta\left(s, \frac{5}{7}\right)+\zeta\left(s, \frac{6}{7}\right)\right) .
$$

Using the functional equation for the Hurwitz zeta functions together with the relation

$$
\begin{aligned}
& \sin \frac{\pi s}{2} \cos \frac{2 j m \pi}{7}+\cos \frac{\pi s}{2} \sin \frac{2 j m \pi}{7}-\sin \frac{\pi s}{2} \cos \frac{2(7-j) m \pi}{7}-\cos \frac{\pi s}{2} \sin \frac{2(7-j) m \pi}{7} \\
& =\sin \left(\frac{2 j m \pi}{7}+\frac{\pi s}{2}\right)-\sin \left(\frac{2(7-j) m \pi}{7}+\frac{\pi s}{2}\right)=2 \sin \frac{2 j m \pi}{7} \cos \frac{\pi s}{2}
\end{aligned}
$$

for $j=1,2,3$, we obtain that, for $\operatorname{Re}(s)<0$,

$$
L_{7}(s, \xi, \rho)=\frac{4 \Gamma(1-s)}{7^{s}(2 \pi)^{1-s}} \cos \frac{\pi s}{2} \sum_{m=1}^{\infty} \frac{1}{m^{1-s}}\left(\sin \left(\frac{2 m \pi}{7}\right)+\xi \sin \left(\frac{4 m \pi}{7}\right)+\rho \sin \left(\frac{6 m \pi}{7}\right)\right) .
$$


Applying the doubling formula for the gamma function and using the fact that

$$
\cos \frac{\pi s}{2}=\frac{\pi}{\Gamma((s+1) / 2) \Gamma((1-s) / 2)},
$$

we get

$$
L_{7}(s, \xi, \rho)=2 \cdot 7^{-s} \pi^{s-1 / 2} \frac{\Gamma(1-s / 2)}{\Gamma(1 / 2+s / 2)} \sum_{m=1}^{\infty} \frac{1}{m^{1-s}}\left(\sin \left(\frac{2 m \pi}{7}\right)+\xi \sin \left(\frac{4 m \pi}{7}\right)+\rho \sin \left(\frac{6 m \pi}{7}\right)\right) .
$$

Comparing the coefficients of the series

$$
\sum_{m=1}^{\infty} \frac{\overline{b(m, \xi, \rho)}}{m^{1-s}} \cdot \Xi(7)
$$

and

$$
\sum_{m=1}^{\infty} \frac{1}{m^{1-s}}\left(\sin \left(\frac{2 m \pi}{7}\right)+\xi \sin \left(\frac{4 m \pi}{7}\right)+\rho \sin \left(\frac{6 m \pi}{7}\right)\right)
$$

we notice that (4.7) implies

$$
L_{7}(s, \xi, \rho)=2 \Xi(7) \cdot 7^{-s} \pi^{s-1 / 2} \frac{\Gamma(1-s / 2)}{\Gamma(1 / 2+s / 2)} \overline{L_{7}(1-\bar{s}, \xi, \rho)} .
$$

This equation is equivalent to (4.8), which completes the proof.

REMARK 2. In the case when $\xi$ and $\rho$ are real numbers satisfying (4.7), it is obvious that $L_{7}(s, \xi, \rho) \in \mathcal{S}_{\mathbb{R}}^{\sharp}$. In this case, the set of equations (4.7) is satisfied for all $(\xi, \rho)$ on the line going through the points $\left(0,-\left(\sin \frac{4 \pi}{7}\right) /\left(\sin \frac{12 \pi}{7}\right)\right)$ and $\left(-\left(\sin \frac{6 \pi}{7}\right) /\left(\sin \frac{12 \pi}{7}\right), 0\right)$.

Remark 3. There exists a $\sigma>0$ such that $L_{7}(s, \xi, \rho)$ has no zeros in the region $\operatorname{Re}(s)>\sigma$.

Indeed, $L_{7}(s, \xi, \rho)$ is a Dirichlet series with bounded coefficients and hence there exists $\sigma>0$ such that

$$
\left|\sum_{m=2}^{\infty} \frac{b(m, \xi, \rho)}{m^{s}}\right| \leqslant \sum_{m=2}^{\infty} \frac{|b(m, \xi, \rho)|}{m^{\operatorname{Re}(s)}}<1 \quad \text { for all } s \text { with } \operatorname{Re}(s)>\sigma
$$

and therefore $\left|L_{7}(s, \xi, \rho)\right|>0$.

ExAmple 3. Define $L_{35}\left(s, \xi_{-}, \chi_{7}\right)=f\left(s, \xi_{-}, \chi_{7}\right)$, where $f$ is defined by $(4.1)$ and $\chi_{7}$ is the principal Dirichlet character modulo 7 , namely,

$$
\chi_{7}(m)= \begin{cases}1, & m \neq \equiv(\bmod 7) \\ 0, & m \equiv 0(\bmod 7)\end{cases}
$$

Writing the function $L_{35}\left(s, \xi_{-}, \chi_{7}\right)$ as a linear combination of Hurwitz zeta functions and proceeding as in the proof of Proposition 4.2 , we see that $L_{35}\left(s, \xi_{-}, \chi_{7}\right)$ belongs to the class $\mathcal{S}_{\mathbb{R}}^{\sharp}$ and satisfies the functional equation

$$
\left(\frac{35}{\pi}\right)^{s / 2} \Gamma\left(\frac{s+1}{2}\right) L_{35}\left(s, \xi_{-}, \chi_{7}\right)=(-1)\left(\frac{35}{\pi}\right)^{(1-s) / 2} \Gamma\left(1-\frac{s}{2}\right) L_{35}\left(1-s, \xi_{-}, \chi_{7}\right) .
$$




\section{Numerical computations}

In this section we present some numerical computations of $\tau$-Li coefficients for different $L$-functions. Our main focus will be the functions from the class $\mathcal{S}_{\mathbb{R}}^{\sharp}$, especially the functions discussed in the previous section. Moreover, since the classical Davenport-Heilbronn function $L_{\mathrm{DH}}\left(s, \xi_{+}\right)$and its associated function $L_{\mathrm{DH}}\left(s, \xi_{-}\right)$can be written as a linear combination of Dirichlet $L$-functions $L(s, \chi)$ and $L(s, \bar{\chi})$, where $\chi$ is the Dirichlet character of modulus 5 such that $\chi(2)=i$, in this section we will also conduct numerical computations related to the function $L(s, \chi)$.

There are a number of approaches to the problem of numerically computing the $\tau$-Li coefficients of a given function. They are based on the different definitions for $\tau$-Li coefficients or on arithmetic formulas for these coefficients. Computationally speaking, the problem is quite demanding, at least for large values of $n$. The main issue is the accumulation of the error terms and the time required for the computation. In order to obtain some meaningful results, high precision is needed in the input. Calculations based on the definition of $\tau$-Li coefficients in terms of sums over zeros of corresponding $L$-functions done with Mathematica 9 with precise error estimates are presented in [7].

In this paper we use Arb, a C library for arbitrary-precision floating-point ball arithmetic, developed by Johansson [8]. It supports efficient high-precision computation with power series and special functions over the real and complex numbers with automatic error control. Thus, the definition of the $n$th $\tau$ - $\mathrm{Li}$ coefficient in terms of the $n$th derivative of the complete $L$-function given in Proposition 3.2, together with the power series representation of the corresponding $L$-function, turns out to be acceptable for calculations.

Moreover, each function we used in the Arb implementation has precision as one of its arguments (variable prec) that is used to control the error of the output. Since the ball arithmetic is used, the output is written in the form of pairs of numbers; the first number is the mid point and the second number is the radius of the interval to which the actual values belong. The resulting error term is equal to the largest radius in the output and hence the size of the error can be easily read off from the data obtained.

We start with the Taylor series representation around a given $\tau$ of the function $\log \left(\xi_{F}(s)\right)$,

$$
\log \left(\xi_{F}(s)\right)=\sum_{k=0}^{\infty} a_{k}(s-\tau)^{k} .
$$

From (3.2), it follows that

$$
\lambda_{F}(n, \tau)=n \sum_{k=0}^{n-1}\left(\begin{array}{c}
n-1 \\
k
\end{array}\right) \tau^{n-k} a_{n-k} .
$$

Thus, the main problem in this approach is to obtain coefficients $a_{k}$ from (5.1). In the present paper we are dealing with the $L$-functions that can be expressed in terms of Hurwitz zeta functions. This allows us to obtain the coefficients $a_{k}$ from the Taylor series representation of Hurwitz zeta functions (a task Arb is especially adept to), combined with the power series representation of the gamma factors that appear in the functional equation.

Extensive sets of numerical data were obtained based on the method explained above. We will present but a select few in this paper. From the output produced we were able to read off the error term. If the error term was not satisfactory, we would increase the value of the variable prec in the arb code in order to reduce the size of the error. For example, in order to produce data presented in Figure 5, we used the variable prec as equal to 20 times the maximal value of $n$ used in calculations. In this way we obtained accurate enough results so that there is no visible difference between the obtained value and the value plus/minus the error term. 
EXAmPLE 4. The Dirichlet $L$-function associated to a Dirichlet character $\chi$ modulo $q$ is defined for $\operatorname{Re}(s)>1$ by the Dirichlet series or the Euler product representation

$$
L(s, \chi)=\sum_{n=1}^{\infty} \frac{\chi(n)}{n^{s}}=\prod_{p}\left(1-\frac{\chi(p)}{p^{s}}\right)^{-1},
$$

and by analytic continuation elsewhere. In the case of the principal character $\chi_{0}$, the corresponding $L$-function can be written in terms of the Riemann zeta function. If the character $\chi$ is not principal, $L(s, \chi)$ is an entire function. The functional equation for the Dirichlet $L$-function is of the form

$$
\left(\frac{q}{\pi}\right)^{(s+\delta) / 2} \Gamma\left(\frac{s+\delta}{2}\right) L(s, \chi)=\frac{\tau(\chi)}{i^{\delta} \sqrt{q}}\left(\frac{q}{\pi}\right)^{(1+\delta-s) / 2} \Gamma\left(\frac{1+\delta-s}{2}\right) L(1-s, \bar{\chi}),
$$

where

$$
\tau(\chi)=\sum_{a \bmod q} \chi(a) e^{2 \pi i(a / q)} \quad \text { and } \quad \delta=\frac{1-\chi(-1)}{2} .
$$

The above-mentioned properties make it abundantly clear that $L(s, \chi)$ belongs to the Selberg class. Furthermore, this Dirichlet $L$-function can be written in terms of Hurwitz zeta functions as

$$
L(s, \chi)=\frac{1}{q^{s}} \sum_{m=1}^{q} \chi(m) \zeta\left(s, \frac{m}{q}\right) .
$$

We will conduct numerical calculations for $L(s, \chi)$, where $\chi$ is the Dirichlet character of modulus 5 with $\chi(2)=i$, that is, the same modulo 5 character that appears in the proof of Proposition 4.1.

In this case, the $L$-function $F(s)=L(s, \chi)$ belongs to the Selberg class $\mathcal{S}$ with $Q_{F}=$ $(5 / \pi)^{1 / 2}, r=1, \lambda_{1}=\mu_{1}=\frac{1}{2}$ and it can be written as

$$
L(s, \chi)=\frac{1}{5^{s}}\left(\zeta\left(s, \frac{1}{5}\right)+i \zeta\left(s, \frac{2}{5}\right)-i \zeta\left(s, \frac{3}{5}\right)-\zeta\left(s, \frac{4}{5}\right)\right) .
$$

In Figure 1, we plot the real parts for the $\operatorname{Li}(\tau=1)$ and $\tau$-Li coefficients for $\tau=2$ attached to $L(s, \chi)$. The results are accurate up to $10^{-500}$. The fact that the values presented in Figure 1 are non-negative and show the absence of oscillations is evidence in support of the generalized Riemann hypothesis for Dirichlet $L$-functions; see also computations presented in [14].

EXAMPLE 5. The Davenport-Heilbronn functions $L_{\mathrm{DH}}\left(s, \xi_{ \pm}\right)$, introduced in Example 1, can be written as the linear combination of two Dirichlet $L$-functions $L(s, \chi)$ associated to the Dirichlet character $\chi$ of modulus 5 with $\chi(2)=i$

$$
L_{\mathrm{DH}}\left(s, \xi_{ \pm}\right)=\frac{1-i \xi_{ \pm}}{2} L(s, \chi)+\frac{1+i \xi_{ \pm}}{2} L(s, \bar{\chi})
$$

as well as a linear combination of four Hurwitz zeta functions, using relation (5.3). Thus, $L_{\mathrm{DH}}\left(s, \xi_{ \pm}\right) \in \mathcal{S}_{\mathbb{R}}^{\sharp}$ with $Q_{F}=(5 / \pi)^{1 / 2}, r=1, \lambda_{1}=\mu_{1}=1 / 2$. The functional equation is given by (4.3) and the complete function is

$$
\xi_{L_{\mathrm{DH}}}\left(s, \xi_{ \pm}\right)=\left(\frac{5}{\pi}\right)^{s / 2} \Gamma\left(\frac{s+1}{2}\right) L_{\mathrm{DH}}\left(s, \xi_{ \pm}\right)
$$

The functions $L_{\mathrm{DH}}\left(s, \xi_{ \pm}\right)$are amongst the first examples of functions which violate the generalized Riemann hypothesis, as noticed in [9] and [2]. However, Bombieri and Ghosh proved 


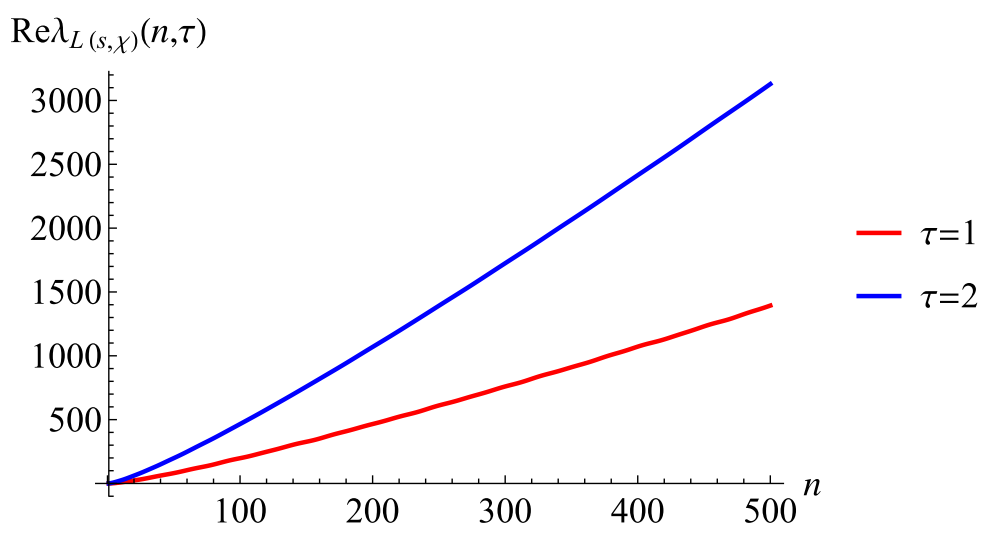

Figure 1. Real parts of $\tau$-Li coefficients attached to Dirichlet L-function.

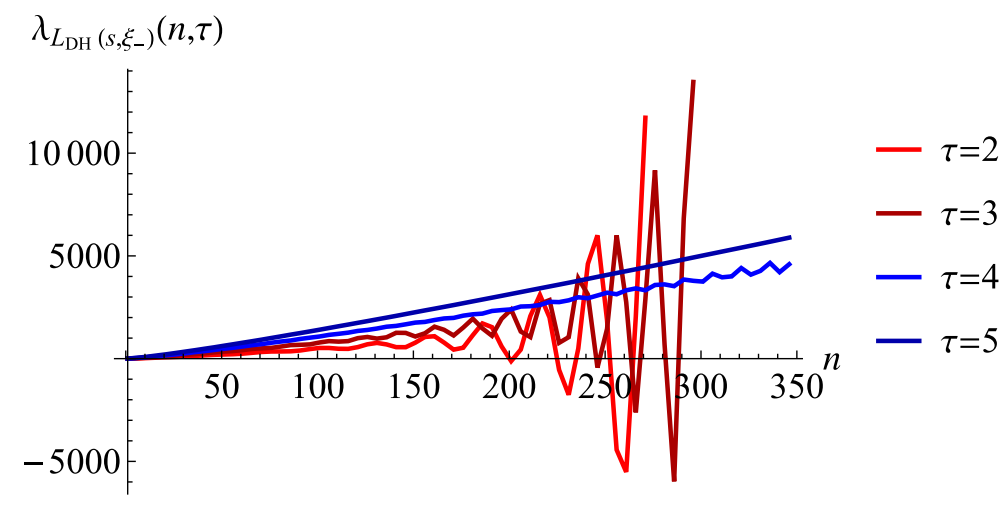

FIGURE 2. $\tau$-Li coefficients attached to $L_{\mathrm{DH}}\left(s, \xi_{-}\right)$.

in [2] that $L_{\mathrm{DH}}\left(s, \xi_{-}\right)$has no zeros in the region $\operatorname{Re}(s)>\sigma_{-}$, where $\sigma_{-} \approx 2.382286$, and that $L_{\mathrm{DH}}\left(s, \xi_{+}\right)$has no zeros in the region $\operatorname{Re}(s)>\sigma_{+}$, where $\sigma_{+} \approx 1.120362$. We have concentrated on the function $L_{\mathrm{DH}}\left(s, \xi_{-}\right)$and have numerically computed $\tau$-Li coefficients for $\tau$ between 2 and 5 with step 0.5 and for $n$ from 1 to 500 with step 5, with accuracy of $10^{-35}$. In Figure 2, we plot some of the data obtained, that is, the $\tau$-Li coefficients for $\tau \in\{2,3,4,5\}$ and $n$ up to 350 . The data for $n$ up to 350 is computed with accuracy of $10^{-110}$. The negative values of $\tau$-Li coefficients and their exponentially growing amplitudes for $\tau=2$ and $\tau=3$ imply the existence of zeros outside the strip $1-\tau / 2 \leqslant \operatorname{Re}(s) \leqslant \tau / 2$ (by Corollary 3.4). This is in complete agreement with the plots of the zeros of the function under consideration given in [2]. The case $\tau=4$ shows the appearance of small oscillations for $n \geqslant 300$, which increase for larger values of $n$, as visible from Figure 3, while the case $\tau=5$ suggests the complete absence of zeros in the right half-plane $\operatorname{Re}(s)>5 / 2$. Again, this agrees with the plot of zeros given in [2], and it reflects the fact that there are zeros of $L_{\mathrm{DH}}\left(s, \xi_{-}\right)$in the half-plane $\operatorname{Re}(s)>2$ and there are no zeros of $L_{\mathrm{DH}}\left(s, \xi_{-}\right)$in the half-plane $\operatorname{Re}(s)>5 / 2$. Better insight into the behavior of the $\tau$-Li coefficients for $L_{\mathrm{DH}}\left(s, \xi_{-}\right)$for a wide range of $\tau$ can be gleaned from the color plot, obtained from the values of $\tau$-Li coefficients for $\tau$ between 1 and 5 with step 0.01 and for $n$ from 1 to 500 with step 1, given in Figure 4. As the $\tau$-Li coefficients can take both very small and very large values, we used a logarithmic scale while preserving the sign. For all values of $\tau$, notice that there is some range of $n$ with increasing value of $\tau$-Li coefficients. 


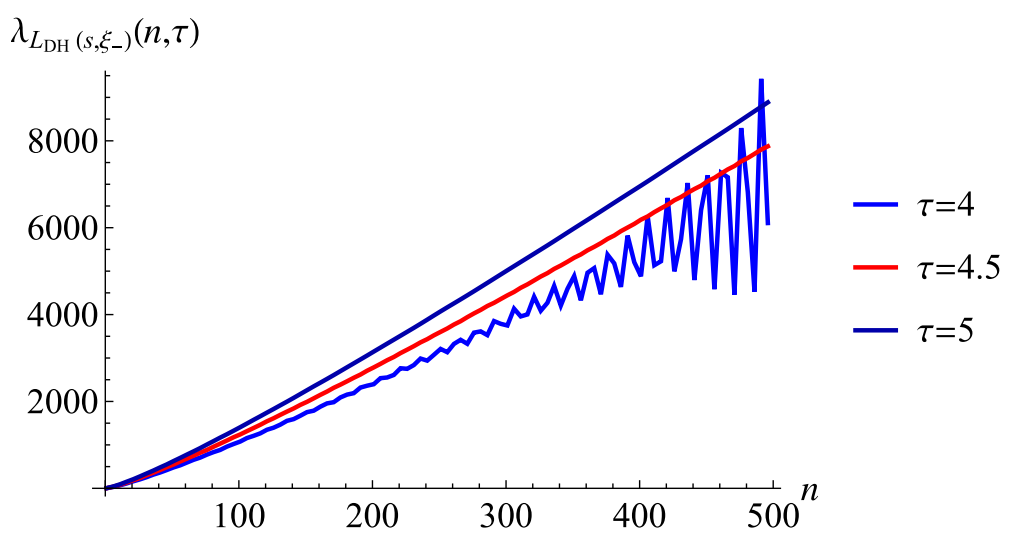

FiguRE 3. $\tau$-Li coefficients attached to $L_{\mathrm{DH}}\left(s, \xi_{-}\right)$.

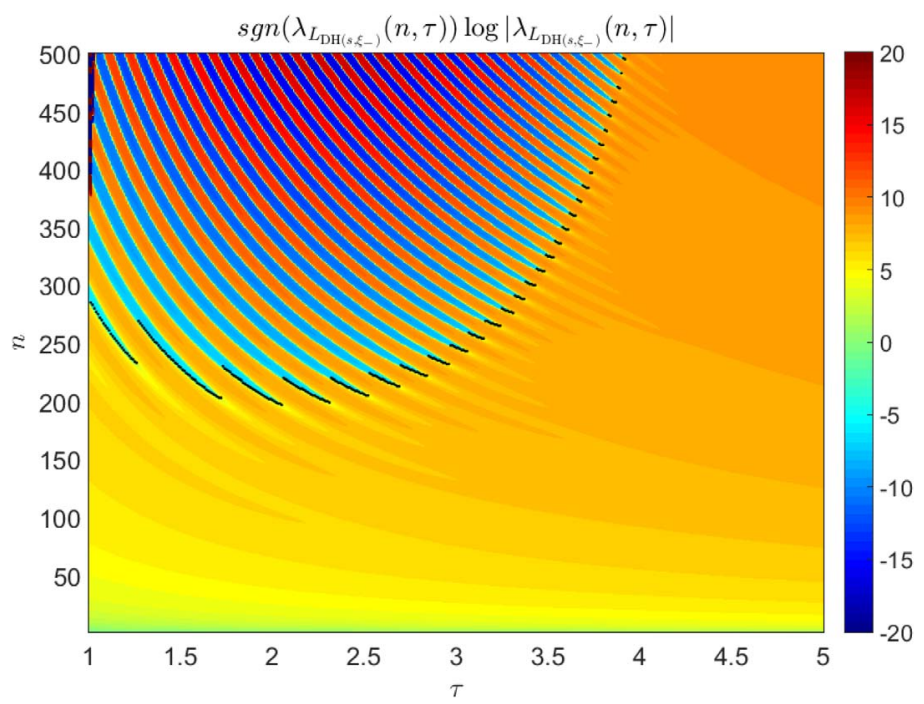

FigurE 4. $\tau$-Li coefficients attached to $L_{\mathrm{DH}}\left(s, \xi_{-}\right)$.

For values of $\tau$ from 1 to nearly 4, notice the gradually increasing oscillations. Alternating blue and red sectors correspond to the ranges of $n$ with negative and positive $\tau$-Li coefficients, while a darker shade indicates the increase in amplitude. The black dots correspond to the smallest value of $n$ for which $\lambda_{L_{\mathrm{DH}}\left(s, \xi_{-}\right)}(n, \tau)$ is negative for a given $\tau$.

EXAMPLE 6 . In this example we consider the function $L_{7}(s, \xi, \rho)$, where $\xi$ and $\rho$ satisfy the system of equations (4.7), and look at the special case when $\xi=2$ and $\rho \approx-3.2469796$. We have computed values of $\tau$-Li coefficients for $\tau$ between 2 and 3 with step 0.2 and for $n$ from 1 to 1000 with step 5 with accuracy $10^{-64}$.

The results are presented in Figures 5, 6 and 7, where we notice appearance of oscillations of $\tau$-Li coefficients for all plotted values of $\tau$. In the case $\tau=2.2$, negative values of the coefficients are obtained, so we may conclude that $L_{7}(s, 2,-3.2469796)$ possesses a zero in the half-plane $\operatorname{Re}(s)>1.1$. The appearance of tiny oscillations for $\tau=3$ suggests also the existence of a zero of $L_{7}(s, 2,-3.2469796)$ in the half-plane $\operatorname{Re}(s)>1.5$. And, indeed, negative values are obtained for values of $n$ close to 5000 as shown in Figure 8. For greater values of $\tau$, 


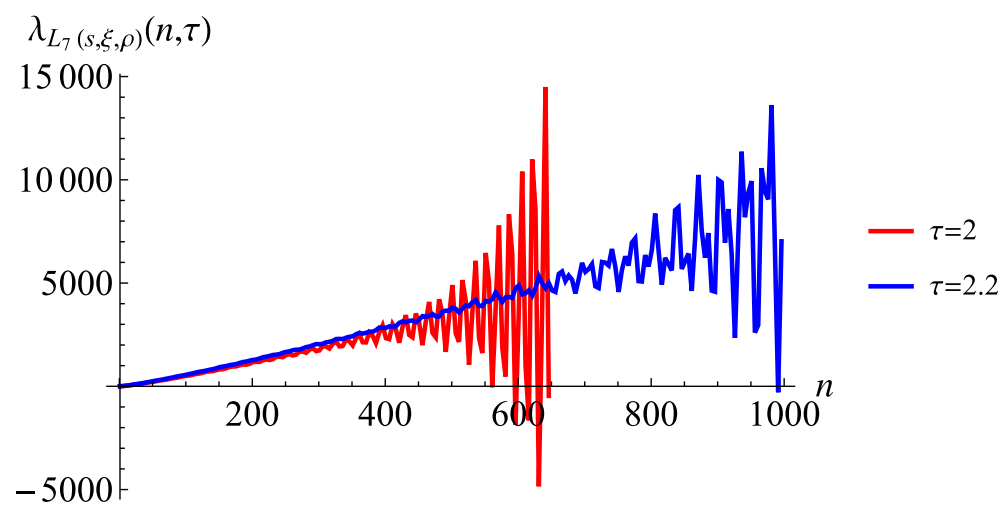

Figure 5. $\tau$-Li coefficients attached to $L_{7}(s, 2,-3.2469796)$.

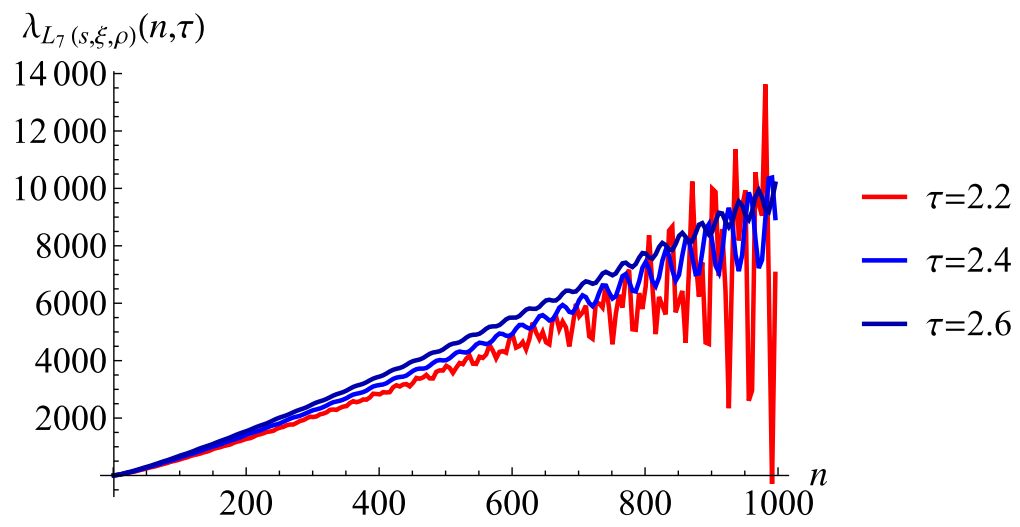

Figure 6. $\tau$-Li coefficients attached to $L_{7}(s, 2,-3.2469796)$.

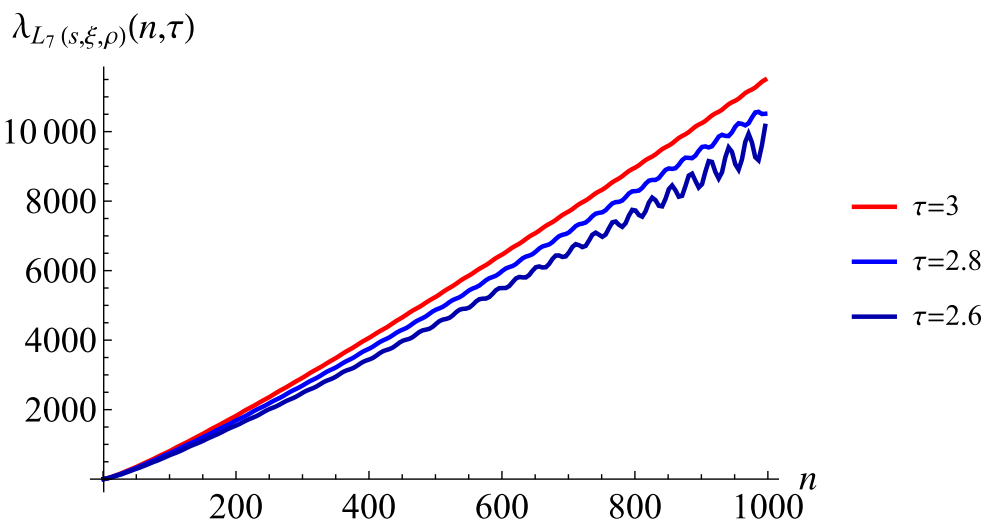

Figure 7. $\tau$-Li coefficients attached to $L_{7}(s, 2,-3.2469796)$.

one would need to compute a wider range of $n$ in order to obtain eventual negative values of $\tau$-Li coefficients. Moreover, a negative value of the $\tau$-Li coefficient is obtained for very large $n$, which also indicates that zeros of $L_{7}(s, 2,-3.2469796)$ with real part bigger than 1.5 are sparse. 


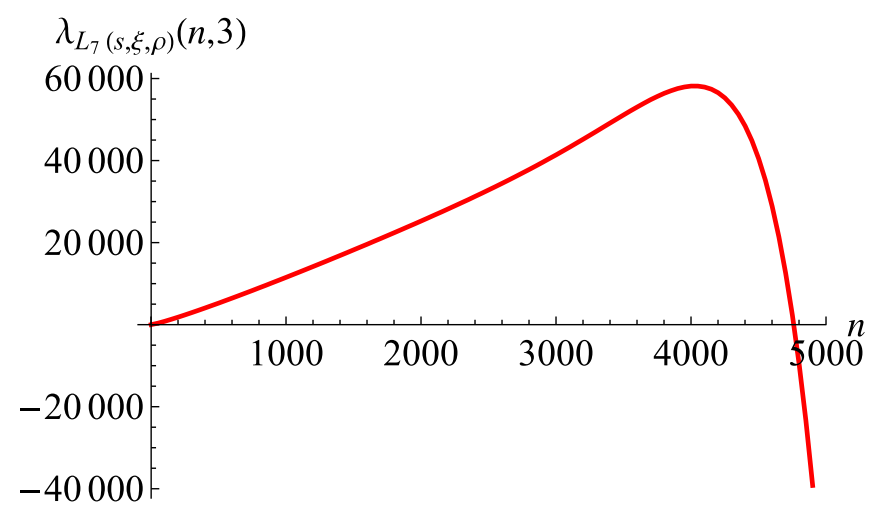

Figure 8. $\tau$-Li coefficients attached to $L_{7}(s, 2,-3.2469796)$ and $\tau=3$.

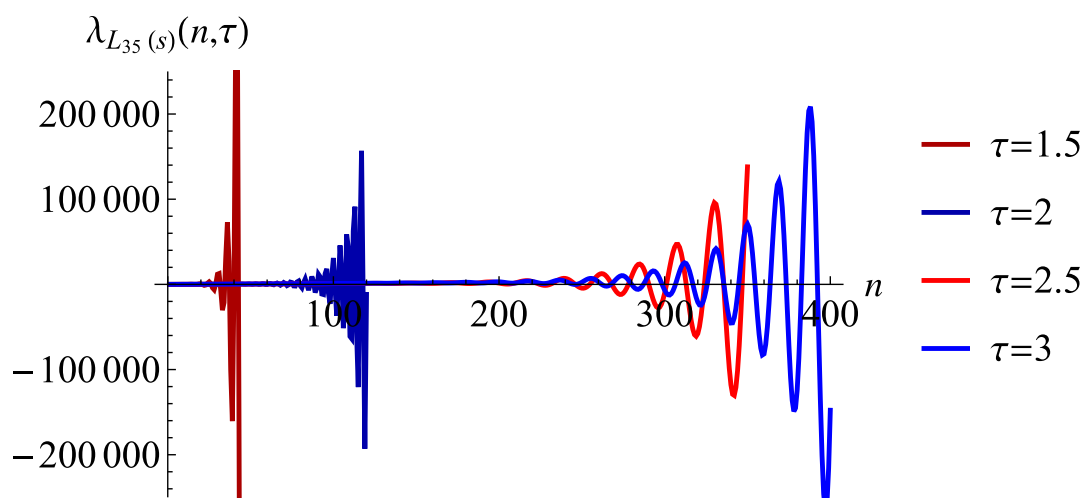

Figure 9. $\tau$-Li coefficients attached to $L_{35}(s)$.

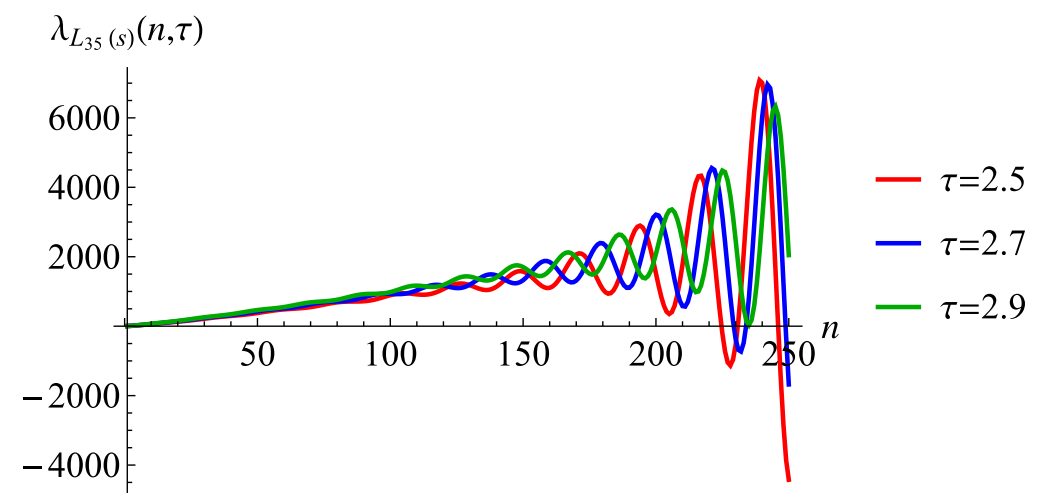

Figure 10. $\tau$-Li coefficients attached to $L_{35}(s)$.

EXAMPLE 7. Let $L_{35}(s)=L_{35}\left(s, \xi_{-}, \chi_{7}\right)$ be the function defined in Example 3. We have computed $\tau$-Li coefficients for values of $\tau$ between 1 and 3 with step 0.01 and for $n$ from 1 to 500 with step 1 , with accuracy up to $10^{-490}$. Some of the data obtained is presented in Figures 9 and 10. 


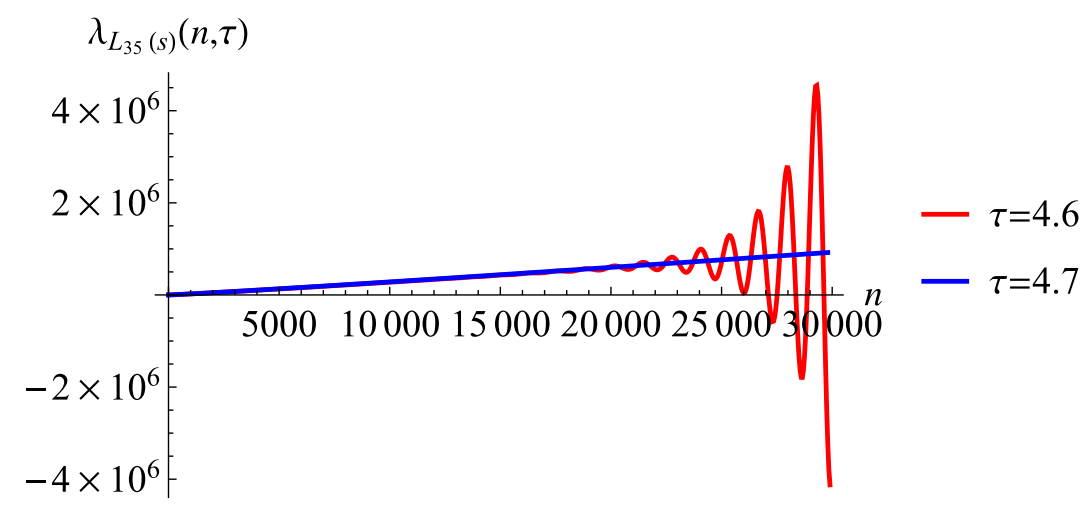

Figure 11. $\tau$-Li coefficients attached to $L_{35}(s)$ and $\tau=4.6$ and $\tau=4.7$.

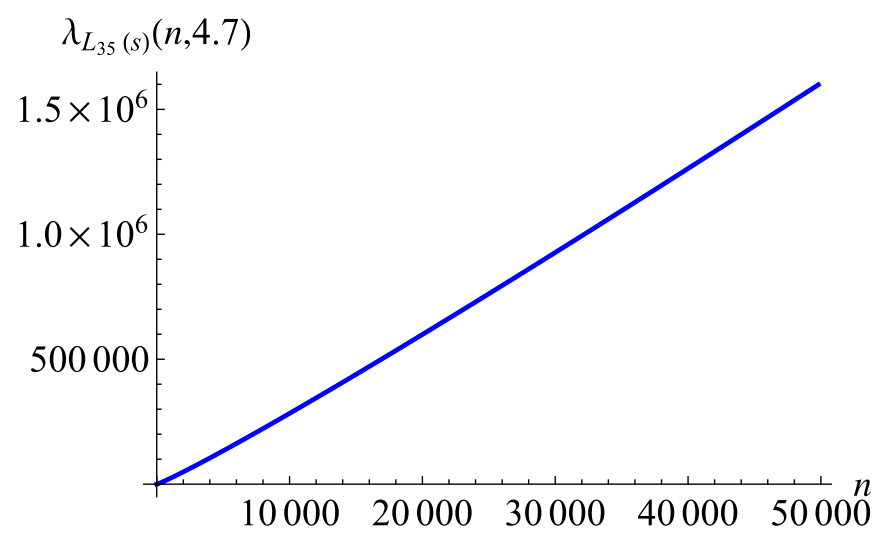

FiguRE 12. $\tau$-Li coefficients attached to $L_{35}(s)$ and $\tau=4.7$.

In [2], it is proved that $L_{35}(s)$ has no zeros in the half-plane $\operatorname{Re}(s)>\sigma_{35} \approx 2.339463$. This means that we should expect the $\tau$-Li coefficients to exhibit oscillations of growing amplitude and eventually take negative values for $\tau<2 \sigma_{35}$ and monotonically increase for $\tau>2 \sigma_{35}$. Figure 11 reflects the change in behavior between $\tau=4.6$ and $\tau=4.7$. Note that, for $\tau=4.6$, at the beginning of the range, it seems that the $\tau$-Li coefficients are increasing, and no oscillations are visible. But later in the range oscillations develop and then they slowly get big enough to produce negative coefficients. In this case, we had to go rather far to see the first negative coefficient. For $\tau=4.7$, we computed the $\tau$-Li coefficients up to $n=50000$ in order to illustrate the increasing behavior, as shown in Figure 12.

All the data we obtained in our numerical calculations, partially presented in the plots above, are in complete agreement with our analytical results and provide numerical evidence for them. They are also in agreement with all other evidence on this topic that we are aware of (for example $[\mathbf{2}, \mathbf{7}, \mathbf{1 2}]$ ). We also produced some new results related to the location of zeros of the function $L_{7}(s, 2,-3.2469796)$, which was not considered elsewhere.

The software we used to obtain the results presented here is quite efficient; thus, we were able to perform the computations in a relatively short time.

Moreover, the above examples clearly indicate that Corollary 3.4 can be interpreted as a method for detecting half-planes containing zeros of certain $L$-functions from $\mathcal{S}_{\mathbb{R}}^{\sharp} \backslash \mathcal{S}$ in the 
sense that the existence of negative values of $\tau$-Li coefficients, computed using the method described above, implies existence of a zero $\rho$ such that $\operatorname{Re}(\rho)>\tau / 2$. For example, Figure 8 proves the existence of a zero $\rho$ of $L_{7}$ in the half-plane $\operatorname{Re}(s)>1.5$. Moreover, the numerical investigations suggest that if $\tau$ - $\mathrm{Li}$ coefficients start oscillating, then it is very likely that for large values of $n$ they will become negative; therefore, once the oscillations are detected one should increase the value of $n$ in order to obtain the negative coefficient. Clearly, there is no numerical method available for proving the zero-free region, since positivity of the first $n \tau$-Li coefficients can only be related to zero-free rectangles of a certain height, which is a function of $n$ and $\tau$. (The numerical evidence suggests that the height of the rectangle is $O(\sqrt{n})$, for large $n$, where the implied constant depends upon $\tau$.)

For the detection of zeros farther from the critical line, we need greater values of $\tau$ and, as our examples suggest, a wider range of $n$ to obtain eventual negative values of $\tau$-Li coefficients. The level of complexity of computations depends obviously on the range of $n$ needed for the detection of a negative coefficient or the approximate position of a negative coefficient. Besides this, our numerical results show that complexity also depends upon the overall distribution of zeros of the $L$-function under consideration, meaning that the level of complexity of computations is not a unique function of $n$ and $\tau$, independent of the $L$-function. For example, for functions similar to the functions of Davenport-Heilbronn type considered in [2], we have noticed that in the case when a large proportion of zeros of $L(s)$ is concentrated close to the critical line (which is the case for example for small positive values of $\xi$ ), then one needs large values of $n$ in order to notice oscillations of $\tau$-Li coefficients for values of $\tau$ very close to 2. Similar observations are discussed in [2], where a search for the zeros is performed using the secant method.

Finally, our method is computationally different from the other methods for searching the zeros with real part bigger than a given number (for example the secant method, used in [2], or the method of deforming Dirichlet series presented in [1]). The method itself is very fast and provides computations with arbitrary precision; however, it is not suitable for computations of zeros; it provides information about (eventual) existence of zeros in certain right half-planes. However, the numerical evidence we obtained suggests that if the first negative $\tau$-Li coefficient is attained at $n$, then in order to speed up the detection of the approximate location of the first zero with real part greater than $\tau / 2$ using the secant method, one should start with initial values $\tau / 2+i \sqrt{n}$ and $\tau / 2+\delta+i \sqrt{n}$, with some small $\delta>0$.

Moreover, the set of data we obtained can be used to investigate additional properties of $\tau$-Li coefficients attached to the functions from $\mathcal{S}_{\mathbb{R}}^{\sharp}$, their zero-free regions or their distribution of zeros. Some additional conjectures could be posed (and hopefully proved) for some specific examples or for a class as a whole. For example, it is interesting to notice that the black dots form a parabolic shape in Figure 4. We believe that the parabolic shape and its position can be described by the zeros of the corresponding function and especially their horizontal distribution. This will be a subject of our future investigations.

\section{Discussion}

The data we present above suggests that the $\tau$-Li coefficients of an $L$-function $F$ in the extended Selberg class with real coefficients $a_{F}(n)$ can exhibit one of two behaviors as we fix $\tau$ and $n$ increases. The first possibility is that they increase at a rate $\sim c_{F} n \log n$, for some constant $c_{F}>0$ depending upon $F$, and exhibit no oscillations. (It is assumed that $c_{F}=(\tau / 2) d_{F}$, where $d_{F}=\sum_{j=1}^{r} \lambda_{j}$ is the degree of $F$; see for example $[4,7]$ or $[\mathbf{1 3}]$.)

The other possibility is that $\lambda_{F}(n, \tau)$ start oscillating at some point. In this case the amplitude of the oscillations keeps growing exponentially, which eventually causes the coefficients to take negative values for some large $n$. Namely, from the formula (3.9), we see that, in the case when the function $F$ possesses a zero in the half-plane $\operatorname{Re}(s)>\tau / 2$, then 
the leading term of the asymptotic behavior of the $\tau$-Li coefficient $\lambda_{F}(n, \tau)$, as $n \rightarrow \infty$, is $-2(1+m)^{n} \sum_{j=1}^{k} \cos \left(n \phi_{j}\right)-l(1+m)^{n}$ for some $m>0$. The sum of cosines can be made arbitrarily close to both $k$ and $-k$ (by Diophantine approximations) and hence this oscillates with amplitude close to $k(1+m)^{n}$ (which grows exponentially).

We surmise that no other behavior is possible for the $\tau$-Li coefficients of a Dirichlet series with real coefficients. Indeed, the same pattern can be seen in the data for the function $L_{7}$ in Example 6. There are really small oscillations for $\tau=3$ in Figure 7, and we found that the $\tau$-Li coefficients become negative later on, which can be seen in Figure 8 . (That is, $\lambda_{L_{7}}(4801,3)<$ $0, \lambda_{L_{7}}(4851,3)<0, \lambda_{L_{7}}(4901,3)<0$ and $\lambda_{L_{7}}(4951,3)<0$.) It will be interesting to see if this pattern extends to the full class of complex meromorphic functions considered in [3].

Acknowledgements. We would like to thank Fredrik Johansson for making Arb available to the world at large, and for making us aware of its existence. We also appreciate the thorough documentation that came with it. We would also like to thank the organizers of the Women in Numbers-Europe workshop, where this project originated. The conference was funded by CIRM, Microsoft Research, Number Theory Foundation, NSF and Clay Mathematics Institute. Their support is gratefully acknowledged. We are grateful to the anonymous referee for his/her careful reading of the manuscript and many valuable suggestions and comments which helped us improve the paper.

\section{References}

1. E. P. Balanzario and J. SÁnchez-Ortiz, 'Zeros of the Davenport-Heilbronn counterexample', Math. Comp. 76 (2007) 2045-2049.

2. E. Bombieri and A. Ghosh, 'Around Davenport-Heilbronn function', Uspekhi Mat. Nauk 66 (2011) 15-66 (Russian); Russian Math. Surveys 66 (2011) 221-270 (English).

3. E. Bombieri and J. C. Lagarias, 'Complements to Li's criterion for the Riemann hypothesis', J. Number Theory 77 (1999) 274-287.

4. A. Bucur, A.-M. Ernvall-Hytönen, A. Odžak, E. Roditty-Gershon and L. Smajlović, 'On $\tau$-Li coefficients for Rankin-Selberg $L$-functions', Women in numbers Europe, Association for Women in Mathematics Series 2 (ed. A. Bucur et al.; Springer International, Switzerland, 2015) 167-190.

5. H. Davenport and H. Heilbronn, 'On the zeros of certain Dirichlet series (second paper)', J. Lond. Math. Soc. (2) 11 (1936) 307-312.

6. A. D. Droll, 'Variations of Li's criterion for an extension of the Selberg class', PhD Thesis, Queen's University Ontario, Canada, 2012; available at http://qspace.library.queensu.ca/jspui/bitstream/1974/ 7352/1/Droll_Andrew_D_201207_PhD.pdf.

7. A.-M. Ernvall-Hytönen, A. Odžak, L. Smajlović and M. Sušić, 'On the modified Li criterion for a certain class of L-functions', J. Number Theory 156 (2015) 340-367.

8. F. Johansson, 'Arb: a C library for ball arithmetic', ACM Commun. Comput. Algebra 47 (2013) no. 4, $166-169$.

9. J. Kaczorowski, 'Axiomatic theory of $L$ functions: the Selberg class', Analytic number theory, C.I.M.E. Summer School, Cetraro, Italy, 2002, Lecture Notes in Mathematics 1891 (eds A. Perelli and C. Viola; Springer, 2006) 133-209.

10. J. Kaczorowski and A. Perelli, 'On the structure of the Selberg class, I: $0 \leqslant d \leqslant 1$ ', Acta Math. 182 (1999) 207-241.

11. J. C. Lagarias, 'Li coefficients for automorphic L-functions', Ann. Inst. Fourier 57 (2007) 1689-1740.

12. K. MaslanKa, 'Li's criterion for the Riemann hypothesis - numerical approach', Opuscula Math. 24 (2004) no. 1, 103-114.

13. K. MAZhoudA, 'On the $\tau$-Li coefficients for automorphic L-functions', Rocky Mountain J. Math., to appear.

14. S. Omar, R. Ouni and K. Mazhouda, 'On the zeros of Dirichlet $L$-functions', LMS J. Comput. Math. 14 (2011) 140-154.

15. N. V. Proskurin, 'On the cubic L-function', St. Petersburg Math. J. 24 (2013) no. 2, 353-370.

16. A. Selberg, 'Old and new conjectures and results about a class of Dirichlet series', Proceedings of Amalfi Conference on Analytic Number Theory (ed. E. Bombieri et al.; Università di Salerno, 1992) 367-385.

17. L. Smajlović, 'On Li's criterion for the Riemann hypothesis for the Selberg class', J. Number Theory 130 (2010) 828-851.

18. E. C. Titchmarsh, The theory of the Riemann zeta-function (Clarendon Press, Oxford, 1951). 
Alina Bucur

Department of Mathematics

University of California at San Diego

9500 Gilman Dr \#0112

La Jolla, CA 92093

USA

alina@math.ucsd.edu

Almasa Odžak

Department of Mathematics

University of Sarajevo

Zmaja od Bosne 35

71000 Sarajevo

Bosnia and Herzegovina

almasa.odzak@pmf.unsa.ba
Anne-Maria Ernvall-Hytönen

Department of Mathematics and

Statistics

Åbo Akademi University

Fänriksgatan 3

$20500 \AA$ Abo

Finland

anne-maria.ernvall-hytonen@abo.fi

Lejla Smajlović

Department of Mathematics

University of Sarajevo

Zmaja od Bosne 35

71000 Sarajevo

Bosnia and Herzegovina

lejlas@pmf.unsa.ba 